\title{
World Economic Reality - Anxiety and Uncertainty of Markets in a Pandemic Context
}

\author{
Alin Ioan CRETU ${ }^{\star}$, Laurentiu TESCAN ${ }^{\star \star}$, George MATEESCU ${ }^{\star \star \star}$, Laurentiu COROBAN ${ }^{\star \star \star \star}$
}

\begin{tabular}{l}
\hline \multicolumn{1}{c}{ A R T I C L E I N F O } \\
\hline Article history: \\
Accepted August 2020 \\
Available online August 2020 \\
\hline JEL Classification \\
A10, I15, I18, J6 \\
Keywords: \\
Global economy, Stress and \\
uncertainty, Pandemic situation, \\
market anxiety, Coronavirus, \\
Everything is "in the fog", Future of \\
the pandemic
\end{tabular}

\section{Introduction}

Economic science - both classical and modern,deeply globalized and theorized - starts from an axiomatic truth, namely that the engine of any economic activity is represented by the consumption of goods and services.Of course this indisputable truth is at least as intuitive as Euclid's axiom in plane geometry.The economy as a scientific and social entity would not have existed if humanity had at its disposal everything it wanted as in the biblical description of the Garden of Eden.It is equally true that the economy would not have existed - at least in the real and usual sense of the word - if the human species did not appear and evolve continuously on this planet.

The functioning of a market economy at any level of complexity, from the smallest to the most extensive, in the following macroeconomic order: local, regional, national, federal, community, continental or intercontinental geopolitical area and respectively global- is more or less negatively influenced and modified by a multitude of parasitic factors with chronic or acute symptoms, of almost any nature and any cause - some of them even hard to imagine and implicitly difficult to predict and especially to counteract later appearance.

In the present case, the almost exponential appearance and spread - globally - of a pandemic generated by a new unknown type of coronavirus, although initially much underestimated by most scientific, economic and especially political and / or governmental elites, has led in time to panic, upheaval and collapse not only in the area of public health but also in the economic area with subsequent extension in all markets in the field of human activities

The retroactive question we all ask ourselves and try to answer is this: "For what essential reasons has the civilized world not been solidly prepared for such a pandemic, and especially once this pandemic has occurred, why has it not been treated? with all due interest, respect and motivation?" Throwing all the blame on China - the probable country of origin of the new coronavirus and / or the WHO, is far from a pertinent and acceptable explanation, although to some extent their initial attitude, which had a lot of hesitation and

\footnotetext{
${ }^{\star},{ }^{\star \star},{ }^{\star \star \star}$ Bucharest University of Economics Studies, Romania, ${ }^{\star \star \star \star}$ West University of Timisoara, Romania. E-mail addresses: cretu.alinioan@yahoo.com (I. A. Cretu - Corresponding author), laurentiu_tescan@yahoo.com (L. Tescan), George.mateescu.vn@gmail.com (G. Mateescu), dorucoroban@yahoo.com (L. Coroban).
} 
especially a namely opacity in communication, negatively influenced the prompt and correct response of the civilized world to the pandemic.

The most logical and acceptable answer to this question seems to be the multitude of semi-false alarms or more correctly the exaggeratedly reactive attitude, in the face of potential pandemics that human society has faced especially in the current millennium.On the one hand, the world perceived the declaration of a pandemic as a decision taken with much, much caution and exaggeration, on the other hand saw these decisions as an information war designed to disrupt the economic and social life of some poles of power to the detriment of others.And last but not least as a ploy of the giant corporations in the pharma industry.The final conclusion at this point is clear: a new aggressive and contagious virus that appeared in the world must be treated from the beginning as an extremely dangerous and risky game, easily assimilated to the famous "Russian roulette".

\section{Literature review}

The specialized economic and political literature from all over the world treats the subject of the present article relatively impartially and especially insufficiently.Usually these references are made especially to the uncertainty of jobs for various fields of activity, small or medium-sized businesses and implicitly to the deep psychological damage, often with deviation in the psychiatric sphere of many people affected in this plan. The continuous and intense stress generated by the anxiety and obsession of losing a job or small or medium business of any kind, ultimately lead to physical and nervous exhaustion. As shown in the paper "Effects of Economic Uncertainty on Mental Health in the COVID-19 Pandemic Context: Social Identity Disturbance, Job Uncertainty and Psychological Well-Being Model" signed by Danijela Godinic, Bojan Obrenovic, Akmal Khudaykulov (2020) published this year-the so-called "loss of individual identity" appears, in the face of this danger that affects his life on all levels, but especially in economic and financial terms, namely in the basic area of human needs, primary needs for individual or family survival.

Other works or impact articles written on this topic highlight the differentiated affect of individuals in society, drawing attention to vulnerable categories of people who may feel much more serious and dangerous this feeling of almost animal fear and total uncertainty about the future and chances of survival (Peterson K Ozili, 2020). Also in this article is an analysis of the mechanisms by which a public health crisis has generated a specific reaction.

In other words, some articles or references in the literature - such as "COVID-19 Impact: Key Takeaways From Our Articles (2020)" draw attention to the projected dimensions for the new and inevitable global economic crisis. "A deeper decline in GDP is now projected this year for most emerging market economies, mainly due to the worsening pandemic and the sharp decline in exports. Our forecast is that the average GDP on the emerging market (excluding China) will contract by $4.7 \%$ this year and then increase by $5.9 \%$ next year. Economic crisis of still difficult proportions for the near future.

Another aspect taken into account by the literature-example: "Coronavirus: Impact on, and Implications for, the Global" in IDC PERSPECTIVE signed by Simon Ellisconsta (2020) is that in the last decade, supply chains have they have moved aggressively to globalized operations, in part to capitalize on cheap labor arbitration opportunities and also to meet the growing demand for products from the same emerging regions.While globalized operations may mean risk diversification, they also expose companies' supply chains to the impact of regional problems, especially where these operations play a significant role globally.

\section{Logical considerations regarding the functioning of the market economy in relatively normal conditions}

Obviously, the functioning of the economy at any level is infinitely more complex than the reduction to an ultra-simplistic concept-consumption.But without necessity, without the economic concept super known and super used by REQUEST, the other basic economic element called SUPPLY has nowhere to appear.Naturally, the two basic economic entities are interconnected and influence each other, automatically leading to the notion of market economy of various natures and sizes - markets that function and selfregulate based on the demand / supply ratio.

As a result of logic and automatic consequence, as the consumption / demand for goods and services increases quantitatively / qualitatively and the pyramid diversifies - the supply will increase and diversify in the same way, pyramidal in turn. And likewise, when the quantitative, qualitative or complex diversified demand decreases, the production and marketing of goods and services will automatically decrease.

\section{Logical considerations regarding the functioning of the market economy in restrictive conditions}

Therefore, regarding both the consumption and the production of goods and services in a system of three-dimensional axes: quantity, quality, diversity - we can say that any major disorder on one of the 3 axes, will generate a dangerous shift of the optimized demand-supply ratio area. Starting from this remark, we can easily guess what would happen when both components of the report will suffer major disorders on all three axes. 
This is exactly the trend of the global economy at the moment.In support of this categorical statement, the supreme justifying element is that almost each of us-more or less palpable, more or less officially-we are simultaneously both suppliers / producers of goods and services and their consumers.And we are all PEOPLE. People who live under the rule of this danger that induces a substantial dose of stress, felt day by day more and more obvious and more and more oppressive.Probably in a short time the cohort of denialists of the existence and / or severity of the virus, who live without major worries, will be narrowed more and more.

This trend will most likely be felt not so directly, generated by the actual risk of severe / critical forms of disease with very high chances of death or long-term deterioration of health, but rather as an indirect effect of a major combined crisis, both economic and social and an equally serious one in the general field of health (the so-called NON-COVID area, extremely heavily hampered and distorted by the extent of COVID disease 19).

\section{Essential characteristics of the distorted functioning of the market economy in a state of emergency / alert, generated by a severe pandemic. Strong elements of stress and uncertainty}

The terrible pandemic situation generated by the appearance and the almost exponential development of the SARS-VOC-2 virus, at planetary level, acts and severely disrupts the quantity, quality and especially the structure / diversity of supply and demand.And this in the conditions in which, at least, the modern civilized and strongly developed economic world has not had a similar experience - from the socalled Spanish flu that appeared more than 100 years ago.It is a period of time that does not seem too long at first glance - but considering the scientific, technological and demographic expansion of human society in this historical period, it becomes huge.

At present, human society is facing an impalpable pandemic danger, almost impossible to assess as a dynamic and gravity in the near future.Because we have before us an enemy about whom we do not know for sure the so-called "zero" patient; the clear and well-determined mode of transmission in the community, the mode of action of the virus and the disorder generated in the infected organism, the pathogenesis of the disease with symptoms often confused.And especially, we do not have at least one effective and efficient treatment as an antidote, but only a total non-specific quasi-treatment based exclusively on the fading of the symptoms of the disease-when this is still possible.In addition, we are forced to blindly believe in the correctness of tests to detect the virus in the body - tests that seem to give a lot of false positive or negative results, increasing confusion and fear.

Of course we do not have a safe and effective vaccine, we like to say that we do not have it for YET, but no one and nothing can guarantee that we will have it before it is too late.

It seems a slightly misplaced and exaggerated statement given that globally, at least 3-4 vaccines are already in the final phase of testing on groups of tens of thousands of people. However, for now, this vaccine is only a hope of humanity - it can remain at this stage of hope, observing what happened in the case of recent previous human epidemics, namely SARS and MERS.

And we can't even estimate how long an immunization with the vaccine will be effective or in its absence with the so-called "herd immunity."Although at the beginning of the pandemic it was scientists who also considered group immunization - in short the mathematical models run on supercomputers have shown that this strategy is rather a utopia or happily the last option of mankind in the fight against this virus.If there are still many people optimistic about the severity of this virus and the pandemic generated, respectively, this view is based on global statistics that do NOT look very worrying at this time, in the sense that in closed cases a score of $95 \%$ discharges or cures compared to only $5 \%$ of deaths compared to about $17,500,000$ cases is a relatively acceptable element, at least for now.

If we add the percentage of only $1 \%$ severe / critical ATI cases compared to about 6,500,000 active cases, the dose of optimism increases - all the more so as all statistics show that the vast majority of people without severe comorbidities or below the limit of 60-65 years old, have extremely low chances of getting severe forms of the disease even if they are infected.But because there is a BUT here, the vast majority of untrained people in the epidemiological sphere and even in the logical analysis of a situation, make a series of essential omissions.

\section{Major omissions in the evolution of the pandemic and implicitly in the evaluation of the necessary measures of medical, political and legislative-administrative nature}

6.1.The virus in its exponential development-uncontrolled by vaccination and by the relentless traversal of tens maybe even hundreds of millions of human organisms more or less genetically diversified, more or less contaminated with other rhinoviruses or coronaviruses- can suffer at a time given extremely dangerous mutations that could drastically change these relationships and why not the beach of vulnerable groups, severely attacking children, young people or relatively healthy adults. This is without taking into account the effectiveness of a potential vaccine that would appear in the near future, a vaccine that could be overtaken by those mutations of the virus. 
This is certainly the most powerful and important reason why the entire scientific world, supported by huge financial funds and state policies focused on medical research and the massive use of artificial intelligence sources, are in a fight against the clock for the development, distribution and widespread use of an effective and relatively safe vaccine in an unlikely short time.

6.2. The mass infection of a human society at a fast pace will practically generate the collapse of national medical systems - especially in countries with deficient systems in terms of quantity and quality.If in such countries we add a relative pandemic preventive indiscipline of the population together with various governmental decisions of relaxation, forced by electoral, economic and / or political pressures, the scenario undoubtedly becomes a reality.Or, this situation, in fact, will generate an avalanche of Covid 19 deaths, uncontrollable and absolutely terrifyingly cynical, through the naturally imposed selection only of those who seem to have chances of survival and not too advanced ages. to be severely affected will simply be sacrificed. And it is not just a prediction but even an indisputable reality observed in countries like Italy, Spain, UK, Sweden, Belgium and many others, countries with medical systems clearly superior to Romania.

But as if it weren't serious enough, another seemingly imperceptible element appears, but with a major destructive effect.When the pandemic collapses the basic and supportive medical systems, when the degree of infection rises sharply - pandemic psychosis will reach its climax. All other non-COVID hospitals, in addition to becoming insufficient, will become extremely vulnerable and ineffective.

The fear of contamination with the new coronavirus will make hospitalization and interventions, including emergency interventions, extremely difficult, involving isolation in the first phase until RT-PCR testing.In addition to the fear of infecting the medical staff and the existing patients in the hospital, there is also the fear of the patient to be hospitalized.For this reason, both doctors and patients will be extremely reserved and susceptible in the procedure of hospitalization, treatment or emergency surgery.

And this situation could in fact generate huge problems in access to hospitalization. Even in the most urgent, affecting equally any patient regardless of age and previous health problems - a case that would lead to unauthorized many preventable deaths in conditions normal.

6.3. Current statistics may suggest a relatively false picture of the severity of the pandemic. This is because the percentage of people discharged does not necessarily mean the percentage of biologically cured patients, but rather those who are negative in the final control tests.It is quite certain that some of those with severe or even moderate forms would present after discharge important sequelae that would affect their health in the future in a rather unpleasant and serious way.

Given the congestion of hospitals, most of them would have extremely difficult access to the postCovid recovery phase. As a result, the real weight of the pandemic may be significantly higher than that resulting from the figures, perhaps correct, but dry and devoid of qualitative significance.

Another statistical element - perhaps even more deceptive in appearance - is the absolute number of patients confirmed positive on testing. This essential information is all the more distorted as access to free RT-PCR tests becomes more selective and focused on people with suspected relatively edifying symptoms in the sense of contacting the virus.

Here, however, the distortion of the real image of the expansion of the infection at the level of a state represents only a secondary element, with the role of pandemic underestimation.

The essential and serious element becomes that many infected individuals remain medically unevaluated and especially remain as a source of undetected and uninsulated community infection - a source all the stronger as epidemiological investigations are less used or less professionally performed by national authorities public health.

On the other hand, the reporting of Covid deaths - according to the case definition given by the WHO can induce by observing the indications and recommendations of this institution very large differences relatively, interstate.Some states practically ignore these definitions of death Covid 19 , formulated by the WHO, considering them aberrantly long pushed to the upper limit, others respect them within more or less reasonable limits, and others make a real excess of zeal, even reporting many suspicious deaths, in the absence of laboratory tests to confirm the presence of the virus.

And probably more damaging than anything in this virtually unequal struggle between humanity and the virus, there is a seemingly strange, seemingly hallucinatory and unlikely element, namely the LACK OF TRUST OF PEOPLE IN GOVERNMENTAL AND EPIDEMIOLOGICAL AUTHORITIES both internally and in mostly externally / globally.

\section{Market anxiety and uncertainty in a pandemic context}

In the face of this gloomy epidemiological picture, unbelievably uncertain and especially deeply stressful and generating anxiety, it is quite easy to intuit a reaction tailored to the markets in all spheres of human activity. And it all starts as we initially pointed out with the sometimes drastic change in the structure and diversity of consumption in a pandemic context, continuing with its global quantitative and qualitative decline.

The drastic change in the structure and diversity of consumption in the existing pandemic context is in fact the massive increase in the basic needs of Maslow's pyramid, namely the fundamental level 1 and 
partially the safety level 2, to the detriment of the other 3 higher levels.Under the given conditions, people limit themselves and will limit themselves to satisfying the physiological and shelter needs and of course the personal and family safety and security.Of course, this is an average trend, without being able to generalize.

Within basic level 1, basic needs can be more or less developed and diversified, depending on age, living environment, minimum disposable income, temperament, various habits and socialization needs, including: personal / family health care; procurement of food, water and medicine; existence and maintenance / rearrangement of a home; the utilities necessary to create an acceptable and at least relatively comfortable microclimate; public or family transport; various other fundamental biological needs; keeping the job and as far as possible a series of activities for recovery and relaxation.

But in a pandemic context, some basic needs will be canceled or blurred, while others that would not seem strictly necessary become practically mandatory, such as access to the Internet and IT equipment, mobile telephony, radio-TV equipment for information.

\section{Maslow's Hierarchy of needs 3D}

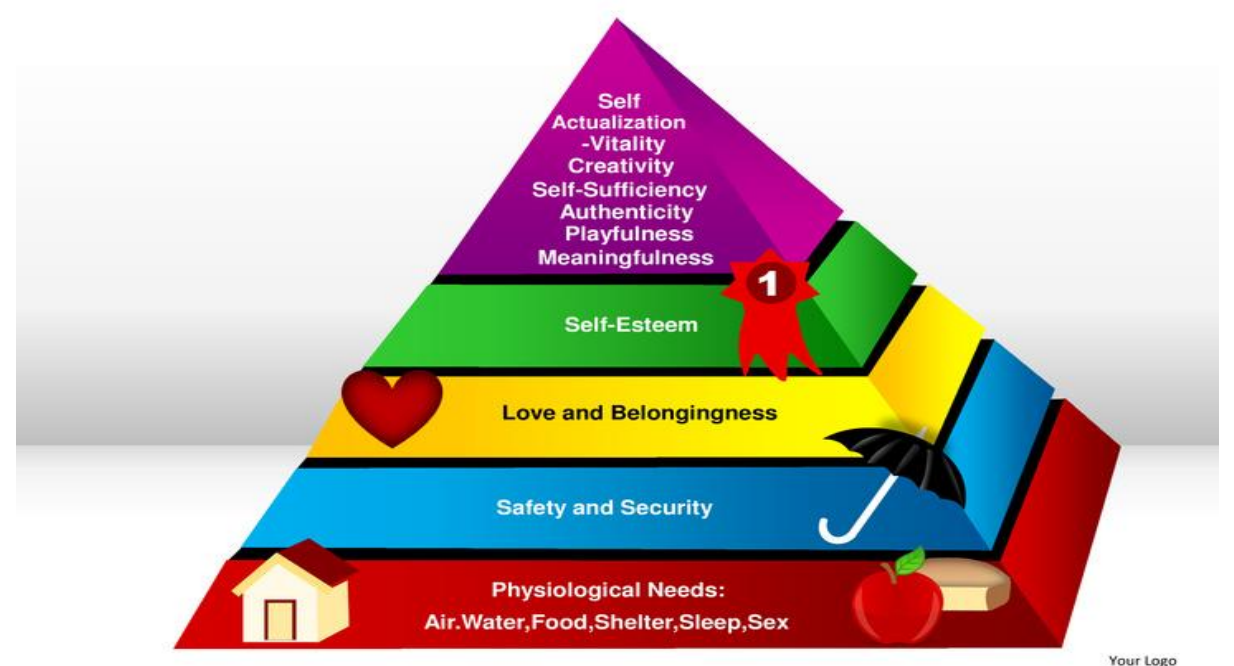

Source: https://www.slideshare.net/PowerPoint-Templates/maslows-hierarchy-of-needs-3d-powerpoint-presentation-templates

Within level 2, many elements related to safety and personal / family security become much less relevant, people limiting themselves to those absolutely vital safety and security needs: personal and family biological safety, job security, business of major importance for family and close people; security of an annuity, pensions, allowances necessary for daily life and others.

Ultimately, it is practically about the safety and security of the means / ways of ensuring the basic needs of the first basic pyramid layer.

Of course, this aspect was extremely clear and obvious in the first phase of the development of the current pandemic, when the isolation / physical distancing caused the so-called lock-down, but in a faded form continues even today.From an economic point of view, activities in multiple fields, such as: domestic and foreign policy; diplomacy; science; the art; culture; sports; army; education / education; entertainmentsocial-administrative were strongly and directly affected by this phenomenon of social isolation.

It can be said that the industry of events of any nature - especially those that cannot be organized outside with safe physical distance; tourism industry and obviously related hotels and restaurants; the entertainment / consumerism industry in almost any form; medical institutions and those intended for education of any kind and at any level have been hit with a huge impact, having simultaneously an indirect effect on all other economic or non-economic activities but generating financial, administrative, educational or medical effects, the list being no far exhaustive.

\section{Final conclusions}

The effect of economic domino, of successive fall in horizontal and vertical chain of an entire and complex socio-economic-financial structure, produced and still produces a huge collapse and obviously had huge effects, in the sense of economic recessions difficult to overcome-both in time and perhaps to a greater extent in terms of costs required.

If we add to this collapse the anxiety and uncertainty of the markets in the near future - as indisputable elements generated by the lack of a perspective and a controllable pandemic evolution - we can intuit how critical the current economic situation is at domestic, community, continental, global level.And all this at a peak of the economy, science and technology of human society and especially in the process of their globalization. 
In fact, it is almost impossible to imagine what our world will look like in the near future - in the absence of the discovery of effective and relatively safe vaccines and / or antidotes for this killer virus - about which we do not yet know if it will fade or become more aggressive. than it is at this time.

It is to be hoped that the intelligence and high experience of specialists in various leading fields in medicine and biology / genetics, hard work logistically / financially supported by governmental and interstate bodies and the overdeveloped technologies of the third millennium, will bring a solution to this in a relatively short time. pandemics, previously effectively unimaginable.

In fact, at least the first phase of pandemic development had an almost mesmerizing effect on people in the developed and civilized world - as if a pandemic, especially of this size, could only appear and develop in the third world, where extreme poverty, lack of medical care and prevention conditions, unsanitary housing and the social environment and especially indiscipline and political and governmental degradation in direct connection with corruption beyond any limits would have tolerated this phenomenon.

All of them, from ordinary citizens to the highest personalities in the political, governmental and last but not least scientific elites, even medical ones, lived with the thought: "Such a thing will not be possible in our democratic, strongly economically developed countries. a sometimes impressive sanitation and especially with a large-scale scientific-medical-epidemiological basis."

This virus has perhaps given us a fully deserved lesson. Let's just hope that we will have the power to overcome this pandemic with minimal losses and that we will draw the conclusions inherent in this harrowing lesson but extremely necessary for the near future of human civilization the planet.

\section{References}

1. Peterson Ozili, Central Bank of Nigeria, Nigeria and ThankomArun, University of Essex, United Kingdom - Spillover of COVID-19 : impact on the Global Economy-March 2020-SSRN Electronic Journal

2. Danijela Godinic, FFZG, Faculty of Humanities and Social Sciences, Zagreb, Croatia;BojanObrenovic, School of Management, Jiangsu University, Zhenjiang, China; AkmalKhudaykulov School of Management, Huazhong University of Science and Technology, Wuhan, China - Effects of Economic Uncertainty on Mental Health in the COVID-19 Pandemic Context: Social Identity Disturbance, Job Uncertainty and Psychological Well-Being ModelInternational - Journal of Innovation and Economic Development-Volume 6, Issue 1, April 2020, Pages 61-74

3. https://www.spglobal.com/ratings/en/research/articles/200204-coronavirus-impact-key-takeaways-from-our-articles-11337257 COVID-19 Impact: Key Takeaways From Our Articles - Comments 20 Aug, 2020 / 16:02Canada, APAC, United States Of America, APAC, EMEA, Latin America

4. https://www.counterpointresearch.com/coronavirus-weekly-update/ - Global Coronavirus Impact and ImplicationsThis version: July 7, 2020 Forthcoming, Review of Economics and Statistics

5. https://www.ilo.org/wcmsp5/groups/public/@dgreports/@dcomm/documents/briefingnote/wcms_745963.pdf - COVID-19 and the world of work. Fourth edition Updated estimates and analysis-27 May 2020; International Laboir Organization

6. https://www.stefamedia.com/abraham-maslow-ierarhia-nevoilor/

7. https://www.worldometers.info/coronavirus/?\#page-top

8. https://www.slideshare.net/PowerPoint-Templates/maslows-hierarchy-of-needs-3d-powerpoint-presentation-templates 